\title{
Electronic structure of a hexagonal graphene flake subjected to triaxial stress
}

\author{
M. Neek-Amal, ${ }^{1,2}$ L. Covaci, ${ }^{1}$ Kh. Shakouri, ${ }^{1}$ and F. M. Peeters ${ }^{1}$ \\ ${ }^{1}$ Departement Fysica, Universiteit Antwerpen, Groenenborgerlaan 171, B-2020 Antwerpen, Belgium \\ ${ }^{2}$ Department of Physics, Shahid Rajaee Teacher Training University, Lavizan, Tehran 16785-136, Iran \\ (Received 13 June 2013; revised manuscript received 26 August 2013; published 23 September 2013)
}

\begin{abstract}
The electronic properties of a triaxially strained hexagonal graphene flake with either armchair or zigzag edges are investigated using molecular dynamics simulations and tight-binding calculations. We found that (i) the pseudomagnetic field in strained graphene flakes is not uniform neither in the center nor at the edge of zigzag terminated flakes, (ii) the pseudomagnetic field is almost zero in the center of armchair terminated flakes but increases dramatically near the edges, (iii) the pseudomagnetic field increases linearly with strain, for strains lower than $15 \%$ but increases nonlinearly beyond it, (iv) the local density of states in the center of the zigzag hexagon exhibits pseudo-Landau levels with broken sublattice symmetry in the zeroth pseudo-Landau level, and in addition there is a shift in the Dirac cone due to strain induced scalar potentials, and (v) there is size effect in pseudomagnetic field. This study provides a realistic model of the electronic properties of inhomogeneously strained graphene where the relaxation of the atomic positions is correctly included together with strain induced modifications of the hopping terms up to next-nearest neighbors.
\end{abstract}

DOI: 10.1103/PhysRevB.88.115428

PACS number(s): 73.23.-b, 73.21.La, 72.15.Qm, 71.27.+a

\section{INTRODUCTION}

Strain engineering can be used to control the electronic properties of nanomaterials. This is of interest for fundamental physics but is also relevant for potential device applications in nanoelectronics. Because the electronic and mechanical properties of an atomic monolayer of graphene are strongly influenced by strain, they have attracted considerable attention over the last years. ${ }^{1-11}$ Uniaxial strain was found to shift or even merge the Dirac cones, ${ }^{2,12,13}$ however, neither uniaxial nor isotropic strain produces a pseudomagnetic field. Such fields can be produced only by inhomogeneous strain which, when applied to graphene, alters the hopping terms between nearest neighbors such that the additional contribution can be seen as a pseudomagnetic field with opposite sign in the two Dirac cones, preserving therefore the time-reversal symmetry. $4,6,8,9,14$ The strain induced modifications of the electronic properties were subsequently confirmed by scanning tunnel microscope (STM) measurements of highly strained nanobubbles that form when graphene is grown on a $\mathrm{Pt}$ (111) surface through the observation of Landau levels. These were shown to correspond to strain-induced pseudomagnetic fields larger than $300 \mathrm{~T}^{7}$ Therefore the study of nonuniform strain distributions at the atomic scale is a promising road for strain engineering purposes, ${ }^{16}$ e.g., the possibility to generate a band gap. In the pioneering work by Guinea $e t$ al., ${ }^{6}$ triaxial strain applied to an hexagonal flake of graphene was shown to induce an energy gap. This is a direct consequence of generating a constant pseudomagnetic field profile at the center of the flake while being variable only at the corners. Recent theoretical studies improved several aspects of the initial theory. ${ }^{9,14,17-20}$ Most of these works concern the strain induced modifications of the continuum low energy Dirac Hamiltonian, which become more complicated as additional orders in strain and momentum are included. ${ }^{14}$ One such important correction is the spatial and strain dependent Fermi velocity. ${ }^{14,15,18}$ This shows that in addition to the pure gauge field given by the first order in strain, additional momentum dependent terms appear. It is also interesting to note that applying triaxial stress on a boron-nitride sheet decreases the energy gap and localizes the frontier orbital in the center of the hexagonal boron-nitride sheet. ${ }^{21}$

By using an atomistic model, we choose both to express the gauge fields in terms of their full tight-binding expression ${ }^{14,19}$ and to describe the electronic properties directly from the tightbinding Hamiltonian with inhomogeneous hopping parameters calculated for the deformed graphene flake.

Furthermore, flakes of graphene can be useful for quantum dot applications. There are several studies that address the latter in terms of the energy spectrum of (unstrained) graphene flakes with different sizes and different edge structures, ${ }^{22}$ within both the continuum model and the tight-binding discrete model for the Dirac equation. ${ }^{23}$ Recently, it was demonstrated experimentally that by straining locally suspended graphene with an STM tip, it is possible to create such quantum dots. ${ }^{11}$ Recently, resonant tunneling through a graphene quantum dot was studied by Qi et al. using triaxially stressed graphene flake. ${ }^{24}$

In this study, we analyze the effect of triaxial strain on the electronic properties of graphene by using an atomistic model which fully takes into account the relaxation of the graphene lattice using bond order interatomic potential. Based on our simulations and using tight-binding theory, we show that several of the predictions of Ref. 6 should be modified resulting in new physical effects.

We found that the vector potential in a hexagonal flake of graphene under triaxial strain behaves differently for zigzag and armchair terminated edges and the corresponding induced pseudomagnetic field is spatially inhomogeneous in both cases. We also show that an energy gap appears in the zigzag hexagon upon applying triaxial strain. This is due to the appearance of pseudo-Landau levels, which form when the pseudomagnetic field is large enough such that the magnetic length is smaller than the flake size. For hexagons with armchair edges, we find that pseudo-Landau levels are absent, due to the small induced pseudomagnetic field in the center, and that the local density of states is enhanced mainly at the edge of the sample. Continuum elasticity theory breaks 


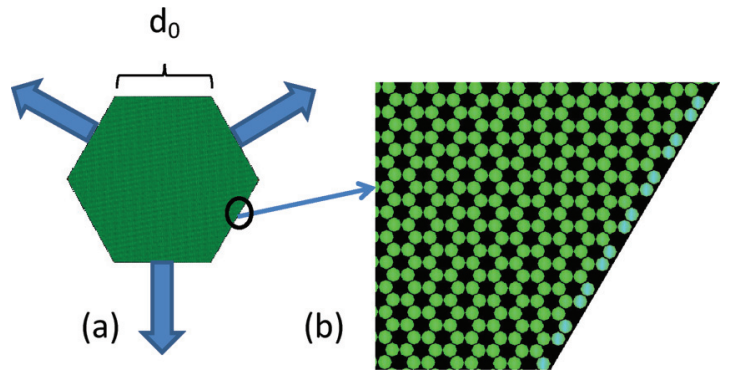

FIG. 1. (Color online) (a) Hexagonal unstrained flake of graphene with armchair edges. The arrows in (a) indicate the triaxial strain directions. (b) The zoomed part of one of the edges.

down at large strains resulting in a nonlinear dependence of the pseudomagnetic field on the strain. We also uncovered a strong finite size effect on the shape and magnitude of the pseudomagnetic field. This also contradicts continuum elasticity theory, which predicts that the field obtained from small flakes can be extrapolated to large ones.

The paper is organized as follows. In Sec. II, we review the theory of elasticity for triaxial strained graphene. In Sec. III, we present our molecular dynamics simulation method for applying triaxial strain on a hexagonal flake. In Sec. IV, the tight-binding model used for calculating the pseudovector potential and pseudomagnetic field is introduced. Section V includes the main results of our work, i.e., lattice deformation obtained from molecular dynamics simulation, vector potential, pseudomagnetic field, and local density of states for both zigzag and armchair flakes.

\section{ELASTICITY THEORY FOR TRIAXIAL STRESS}

Figure 1(a) shows a hexagonal graphene flake having armchair edges with side size $d_{0}$. The blue arrows indicate the triaxial stress directions, which are along the three equivalent crystallographic directions. In Fig. 1(b), the edge structure is shown. In polar coordinates $(r, \theta)$, the applied triaxial stress results in a displacement vector $\vec{U}=\left(U_{r}, U_{\theta}\right)=$ $C r^{2}(\sin (3 \theta), \cos (3 \theta))$, where $C$ is a constant determining the strength of the applied stress which has the dimension of inverse length. ${ }^{6,9}$ The displacement can be written in Cartesian coordinates as

$$
\vec{U}=C\left(2 x y, x^{2}-y^{2}\right) .
$$

On the other hand, linear elasticity theory for an isotropic material leads to the stress-strain relation $\sigma_{j k}=\lambda \delta_{j k} u_{j j}+$ $2 \mu u_{j k}$, where $\lambda$ and $\mu$ are the Lamé parameters that determine the stiffness of a material and $u_{j k}$ are elements of the strain tensor. If we substitute $\vec{U}$ in Eq. (1), the components of the stress tensor in Cartesian coordinates can be found as

$$
\sigma(x, y)=4 \mu C\left(\begin{array}{cc}
y & x \\
x & -y
\end{array}\right),
$$

where the $x$ axis is taken along the zigzag direction and the $y$ axis along the armchair direction. Here, both zigzag and armchair hexagonal graphene flakes are studied separately. Notice that the three stressed edges (and also the free edges) should have only one type of edge: zigzag or armchair. The zigzag hexagonal flake is not shown in Fig. 1. In the following, we present the methodology and compare our atomistic simulation results with those predicted by the above simple continuum elasticity theory.

\section{MOLECULAR DYNAMICS SIMULATION WITH TRIAXIAL STRAIN}

We apply triaxial stress on the edges of a hexagonal flake of graphene as indicated in Fig. 1. Two different samples with armchair and zigzag edges are considered and consist of 40234 and 40016 carbon atoms, respectively, with optimized initial side, i.e., $d_{0}=20 \mathrm{~nm}$ (the size effect is studied in Sec. V C). When applying stress, all atoms in the three nonconsecutive edges experience external constant force during each molecular dynamics simulation (MD), which causes them to move along the direction of the arrows shown in Fig. 1(a). The system reaches its equilibrium size under the applied force, resulting in different strains for different forces. Notice that one can fix the boundary atoms and move them with constant velocity along the direction perpendicular to the edges, however, this will result in a larger pseudomagnetic field (see Appendix C). All results of the present work are obtained using the force constant method. In our MD simulations, we have used the adaptive intermolecular reactive bond order (AIREBO) potential, ${ }^{25}$ which is, in particular, suitable for simulating hydrocarbons. The stretching process is done at low temperature (namely, $T=10$ $\mathrm{K})$. After reaching the desired strain (i.e., we study strains up to the breaking point), we performed energy minimization to find the minimum energy configuration using the conjugate gradient method under the constant force condition. We notice that there is no out-of-plane deformation in the final minimized samples. As a measure of strain, we used $\epsilon=\left(d-d_{0}\right) / d_{0}$, where $d$ is the distance between the center of the hexagonal flake and one of the edges under stress. The final strained samples are no longer perfect hexagons (see Fig. 3). The stress tensor is obtained from the interatomic many-body interaction between the carbon atoms using the method employed in LAMMPS ${ }^{26}$ which includes different energy contributions in the AIREBO potential. Therefore our MD results for the stress elements are not restricted to the simple linear relation between stress and strain.

The applied stress changes the reciprocal lattice as well as the real-space lattice. Using the two-dimensional Fourier transformation of the final strained samples the change in the reciprocal lattice (Brillouin zone) is obtained. Figures 2(a)2(c) show the diffraction pattern of the original unstrained,
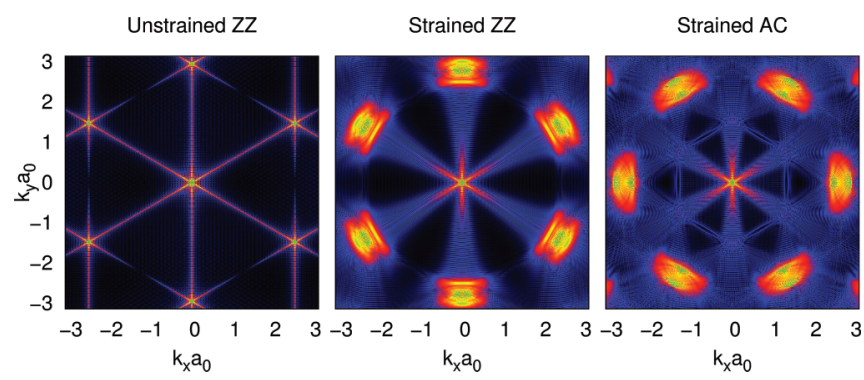

FIG. 2. (Color online) Diffraction pattern for (a) undeformed hexagonal flake (b) zigzag (c) and armchair hexagonal flakes under triaxial stress. 
strained zigzag, and armchair flakes, respectively, where $\epsilon=$ $13 \%$. Notice that the original $K$ and $K^{\prime}$ valleys are altered differently due to the in-plane triaxial strain. The variation in the diffraction pattern is different from that of corrugated suspended graphene due to intrinsic thermal ripples. ${ }^{27}$ It is expected that such new patterns can be realized in experiment. The direct lattice and the reciprocal lattice deformations alter the hexagonal shape of the original Brillouin zone and will change the vector potentials. ${ }^{17}$

\section{TIGHT-BINDING MODEL FOR THE GAUGE FIELD}

The electronic properties of graphene are described by a tight-binding Hamiltonian for the $\pi$ carbon orbitals. We employ the Hamiltonian that describes the low-energy band structure $^{3}$ ignoring the spin degrees of freedom, which is motivated by the fact that no spin-flipping terms are present in the Hamiltonian. Strain is included in the modified hopping amplitudes between the $\pi$ orbitals, $t_{\pi}\left(r_{i j}\right)$, according to the empirical relation $t_{\pi}\left(r_{i j}\right)=t_{0} e^{-\beta\left(\left|\vec{\delta}_{i j}\right| / a_{0}-1\right)}$, where $\beta=-3.37$, $t_{0}=2.7 \mathrm{eV}, a_{0}=1.42 \AA$ is the equilibrium intercarbon distance, and $\vec{\delta}_{i j}$ is the vector that connects the two neighboring atoms in the strained sample; here, we consider both nearest and next-nearest neighbor terms. All neighbor distances are obtained from the relaxed MD sample. Since the applied strain is in plane, the effect of misalignment of the $\pi$ orbitals resulting from the finite curvature is negligible. More details of the used tight-binding model and the related numerical techniques for performing large system calculations can be found in our previous works. ${ }^{16,28-30}$ From a theoretical point of view, external forces deform graphene so that the nearest-neighbor distances become nonequal and results in modified hopping parameters, which are now a function of the atomic positions $t(\mathbf{r}){ }^{3,31}$ The Fermi surface is displaced in reciprocal space $\left(\vec{k} \rightarrow \vec{k}-\frac{e}{\hbar} \vec{A}\right)$, where $\vec{A}$ is the fictitious vector potential and $\vec{k}$ refers to the $K$ point and, consequently, a pseudomagnetic field $\left(\vec{B}=\frac{1}{e v_{F}} \vec{\nabla} \times \vec{A}\right.$, where $e$ is the unit of charge and $v_{F} \sim$ $10^{6} \mathrm{~m} / \mathrm{s}$ is the Fermi velocity in graphene ${ }^{3}$ ) appears. ${ }^{7}$ The new term should be added to the original tight-binding Hamiltonian due to the modification of the hopping parameters, which includes the induced gauge field:

$$
A_{x}+i A_{y}=\sum_{\vec{\delta}_{a b}} \delta t_{a b}(\vec{r}) e^{-i \vec{K} \cdot \vec{\delta}_{a b}},
$$

where $\delta t_{a b}=t-t_{0}$ is the difference between the hopping parameters of the deformed and the original lattices. The position of the $\vec{K}$ valley in the original lattice is $\vec{K}=\frac{4 \pi}{3 a_{0} \sqrt{3}}(1,0)$ if the $x$ axis is taken along the zigzag direction. We use Eq. (3) to calculate the pseudomagnetic field, which includes both changes in the hopping parameters and lattice deformations. As recently shown in Ref. 32, this is the correct way to extract the pseudomagnetic field from atomistic simulations.

Notice that one can use the displacement vectors given by Eq. (1) for producing a deformed hexagonal flake without molecular dynamics simulation relaxation. The latter can be used as the input coordinates for calculating the gauge field and the corresponding pseudo magnetic field. However, we show in Appendix A that the resulting Landau levels and the obtained lattice deformations are not consistent with those
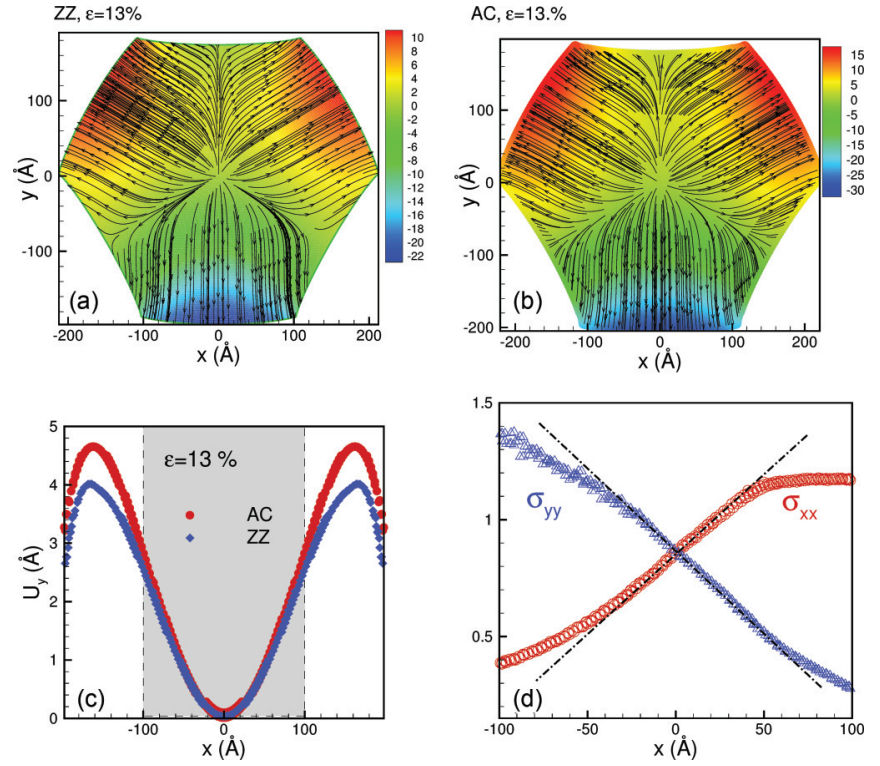

FIG. 3. (Color online) The displacement vector pattern in strained (a) zigzag (b) and armchair hexagonal graphene flakes. The variation of (c) $U_{y}$ vs $x$, where $|y|<2 \AA$ for zigzag (circles) and armchair (triangles) hexagonal flakes subjected to an inhomogeneous strain of about $\epsilon=13 \%$. In (d), the two diagonal elements of the stress tensor, i.e., $\sigma_{x x}$ and $\sigma_{y y}$ are compared to the prediction from elasticity theory (dashed lines), where $|y|<2 \AA$, see the text.

found here after fully relaxation of the coordinates using the true relaxation mechanism of the atomistic system. Since the magnitude of the pseudomagnetic field involves a summation over the nearest neighbors, different lattice orientations with respect to the applied forces will give different pseudomagnetic fields. For example, Eq. (1) gives a constant pseudomagnetic field for a zigzag flake and no field for an armchair one. $6,14,19$

\section{RESULTS}

\section{A. Lattice deformation}

In Fig. 3, we show the lattice deformation due to triaxial strain (with $\epsilon=13 \%$ ) in zigzag terminated (a) and armchair terminated (b) hexagonal flakes, respectively. The arrows indicate the displacement vector streamlines, i.e., the vector field $\vec{U}$. In both cases, the field refers to the triaxially stressed systems. The color code refers to the value of the component of the displacements $U_{y}$, i.e., positive (negative) displacement is indicated by red (blue) color without any out-of-plane movements. The displacement vectors in zigzag and armchair flakes are similar but the electronic properties will be rather different. The streamline vectors are perpendicular to the three stressed edges and are almost parallel to the free arc-shape edges. The larger the strain the larger the concavity of the edges.

In order to compare our numerical results with those predicted by Eq. (1), we plot the $U_{y}$ components of the displacements from our MD simulations and the two main components of the stress tensor in panels (c) and (d) of Fig. 3. In Fig. 3(c), we show the variation of $U_{y}$ with $x$ for constant $y$ (i.e., $|y|<2 \AA$ ) in the strained armchair (blue diamonds) and zigzag (red circles) graphene flake, which were subjected 
to the strain $\epsilon=13 \%$. There is a clear deviation from linear behavior [see $U$ in Eq. (1)], which is very pronounced close to the borders of the sample. The same behavior is found for $U_{x}$ but is not shown. In Fig. 3(d), we show the variation of $\sigma_{y y}$ and $\sigma_{x x}$ with $x$ for a typical strain (13\%). The lines indicate the stress component from Eq. (2). It is again seen that there is considerable deviation from the prediction of continuum elasticity theory [see Eq. (1)], particularly beyond $|x|>50 \AA$ where the zigzag and armchair profiles deviate from each other; notice that linear elasticity theory does not distinguish these two different lattice orientations. The reason for these deviations is the effect of the free edges on the lattice distortion that results in a complex strain distribution. The latter effect was neglected in the previous studies. ${ }^{6,9}$ Nevertheless, in most recent theoretical works, the main attention was directed to the center of the flake which as we see from Figs. 3(c) and 3(d), for the region $|x|<50 \AA$, agrees with elasticity theory.

\section{B. Pseudomagnetic field}

Using Eq. (3), we calculated the vector potential for the obtained lattice deformations from our MD simulations. It is interesting that the vector potential streamlines exhibit neither constant nor circular orbits. In the zigzag flake, the vector potential shows orbits having deformed triangular shape [there is a kind of threefold symmetry, see Fig. 4(a)] but surprisingly for the armchair flake, they do not exhibit orbits and the vectors follow an hyperbolic function, see Fig. 4(b). Therefore the corresponding pseudomagnetic field for the two cases will be very different. The pseudomagnetic field profiles as generated by the strain configurations are shown in Figs. 4(c) and 4(d) for the zigzag and armchair flakes, respectively. The important effect is the variation of the field over the zigzag flake, specially around the center of the flake (see Appendix B). For the armchair flake, the induced magnetic field is close to zero and varies smoothly in the central part, see Fig. 4(d). For better visualization, we plot in Fig. 4(e) the pseudomagnetic field along the $x$ axis of Figs. 4(c) and 4(d). It is interesting to note that the pseudomagnetic field in the zigzag flake exhibits threefold symmetry while for the armchair it shows a more complex pattern. We also present in Fig. 4(f) a comparison between the central pseudomagnetic fields obtained directly from the deformation, the field obtained from the electronic gap between the zeroth and first pseudo-Landau levels, the prediction from Ref. 6, and the result using Eq. (1), i.e., $B=16 C \hbar / a_{0} e$. A linear regime is found only for $\epsilon<15 \%$, while beyond this value, the pseudomagnetic field behaves nonlinearly with respect to $\epsilon$. The values obtained from the deformation and electronic properties are very similar but much smaller than the prediction of continuum elasticity theory [triangular symbols in Fig. 4(f)]. We attribute this discrepancy to the change in the lattice structure due to the relaxation of the graphene sheet. We note that for the same $\epsilon$ the shape of the flake obtained from the MD simulation is very different from the one obtained from the deformation defined by Eq. (1).

\section{The size effect in pseudomagnetic field and the LDOS}

In order to study the size effect on the pseudomagnetic field, we performed several additional MD simulations for
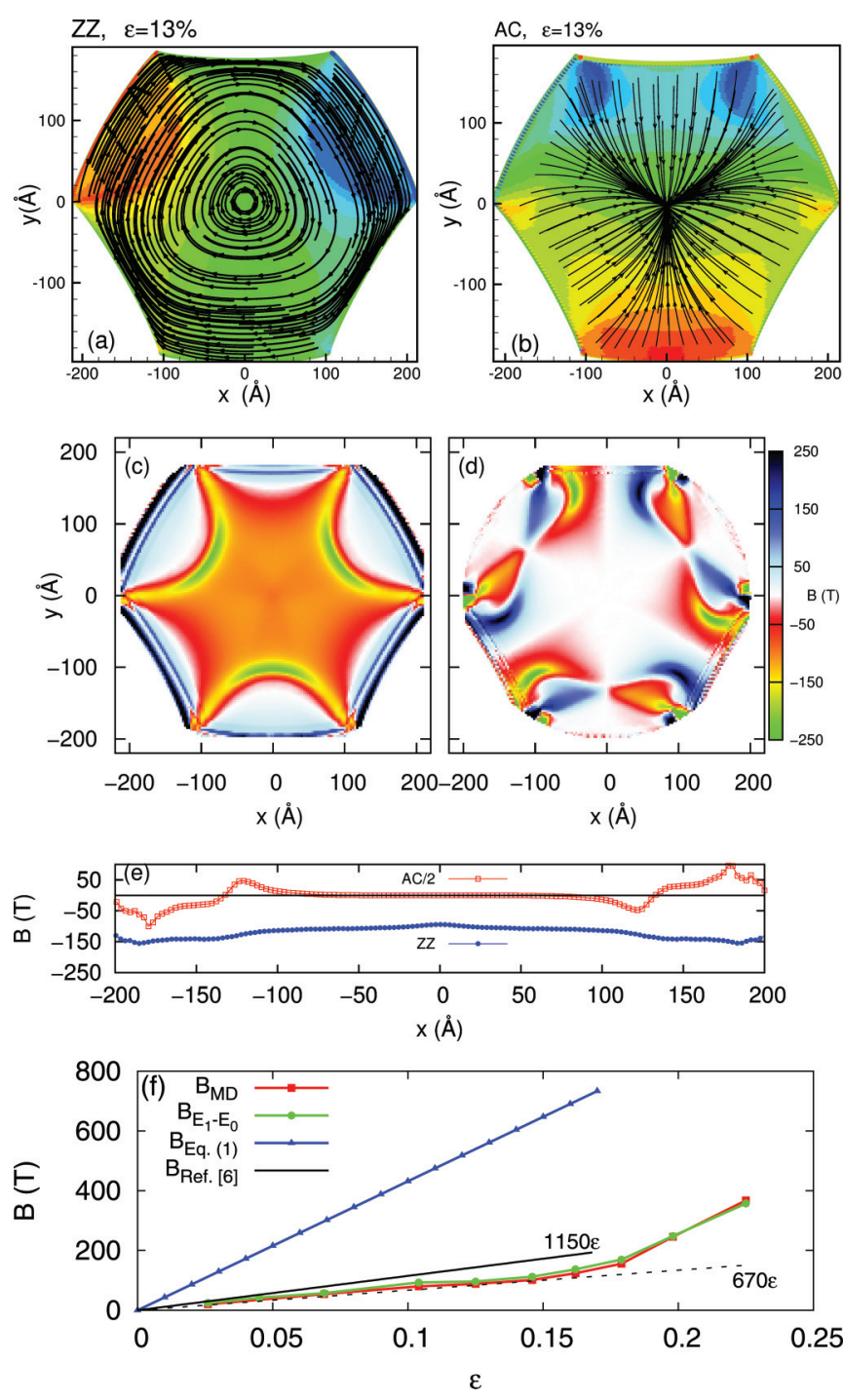

FIG. 4. (Color online) Vector potential $\vec{A}$ for strained hexagonal zigzag (a) and armchair (b) graphene where $\epsilon=13 \%$. The corresponding pseudomagnetic field is shown in (c) for zigzag and (d) armchair flakes. (e) The pseudomagnetic field profile along the $x$ axis for both armchair (red square) and zigzag (blue cricles) flakes. (f) Comparison between the pseudomagnetic field at the center of the zigzag flake obtained from the deformation (red squares), from the electronic gap between the zero and first pseudo-Landau levels (green circles), the prediction from linear elasticity theory [Eq. (1)] (blue triangles) and the results given by Ref. 6 (black straight line).

hexagonal zigzag flakes with $d_{0}=12.6,18$, and $80 \mathrm{~nm}$ in addition to the one discussed in detail, i.e., $d_{0}=20 \mathrm{~nm}$. The largest flake that we studied has around 633750 atoms. In Fig. 5, we show the pseudomagnetic field profile for different sizes but for the same strain $\epsilon=17.9 \%$. We observe that the field profile cannot be extrapolated from small size to large sizes, since in small flakes, the edge effects are larger resulting in very inhomogeneous fields near the center of the hexagon. The linear elasticity theory predicts that the field should inversely be proportional to the size, however, as we will discuss in Appendix A, the larger the system the more (nonlinear) deviation of lattice deformation given by Eq. (1) from that of we obtained from MD lattice relaxation. 


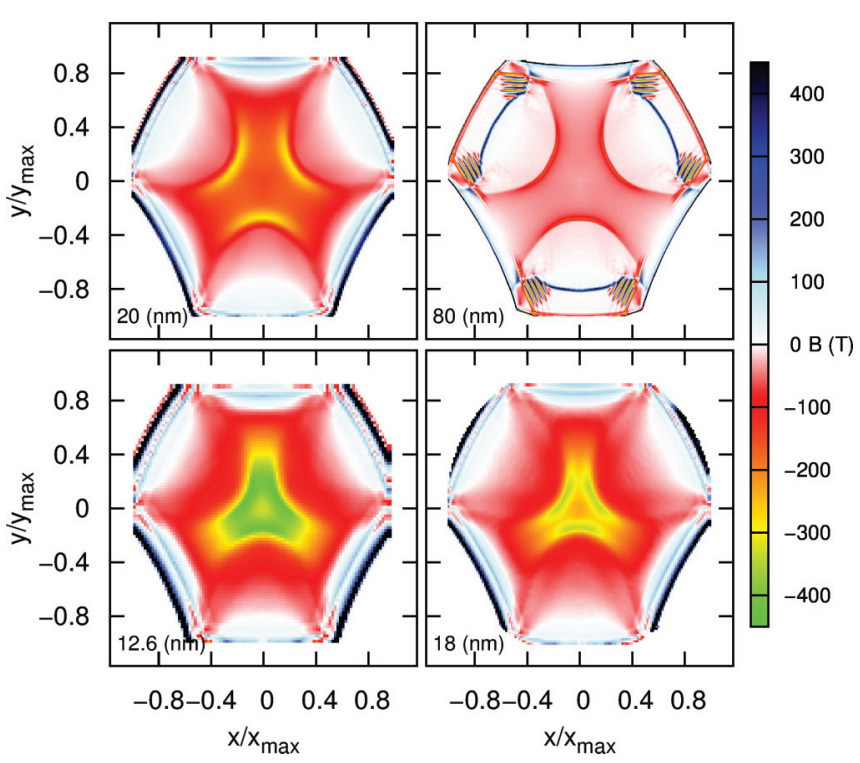

FIG. 5. (Color online) Pseudomagnetic field profile for different hexagonal flakes of sizes $d_{0}=12.6,18,20,80 \mathrm{~nm}$ where $\epsilon=17.9 \%$. The axes were scaled by $x_{\max }$ and $y_{\max }$ in order to have a direct comparison of the profiles.

The latter affects pseudomagnetic field as well. Another source for such a nonpredictable relation between the size and pseudomagnetic field is the absence of dangling bond in the continuum plate theory. In MD simulation relaxation, the free boundaries increase the energy of the system and affects the stress distribution more complicate for the larger system. We did not find any specific role for the relation between pseudomagnetic field and size of our systems.

\section{Local density of states}

In order to investigate the effect of strain on the electronic properties, we input the relaxed atomic positions obtained from our atomistic simulation into a real-space tight-binding model. Our main focus in this work is to find the LDOS maps, which could be directly accessed by STM experiments. The LDOS maps are obtained by expanding the Green's function at each atomic position in terms of Chebyshev polynomials. The details of this expansion can be found in our previous works. ${ }^{28-30}$ Here, we use both nearest neighbor and next-nearest neighbor contributions in the tight-binding Hamiltonian in order to account both for the strain induced vector potential (nearest neighbor) and the strain induced scalar potential (next-nearest neighbor). First, in Fig. 6(a), we show the LDOS for an atom in the A sublattice (which are under stress at the three edges) in the center of the hexagon for various applied force strengths. Several interesting effects can be observed. Because we also include next-nearest neighbor hopping amplitudes in the calculation, the Dirac point for the unstrained hexagon sits at a finite energy, $E_{D}=3 t^{\prime}$, where $t^{\prime}$ is the next-nearest neighbor hopping amplitude. We therefore shift all the LDOS curves such that the Dirac point of the unstrained configuration sits at the Fermi level. We also observe an additional shift for the strained configurations because of the exponential suppression in $t^{\prime}$, which could be understood in terms of a strain induced scalar potential, which
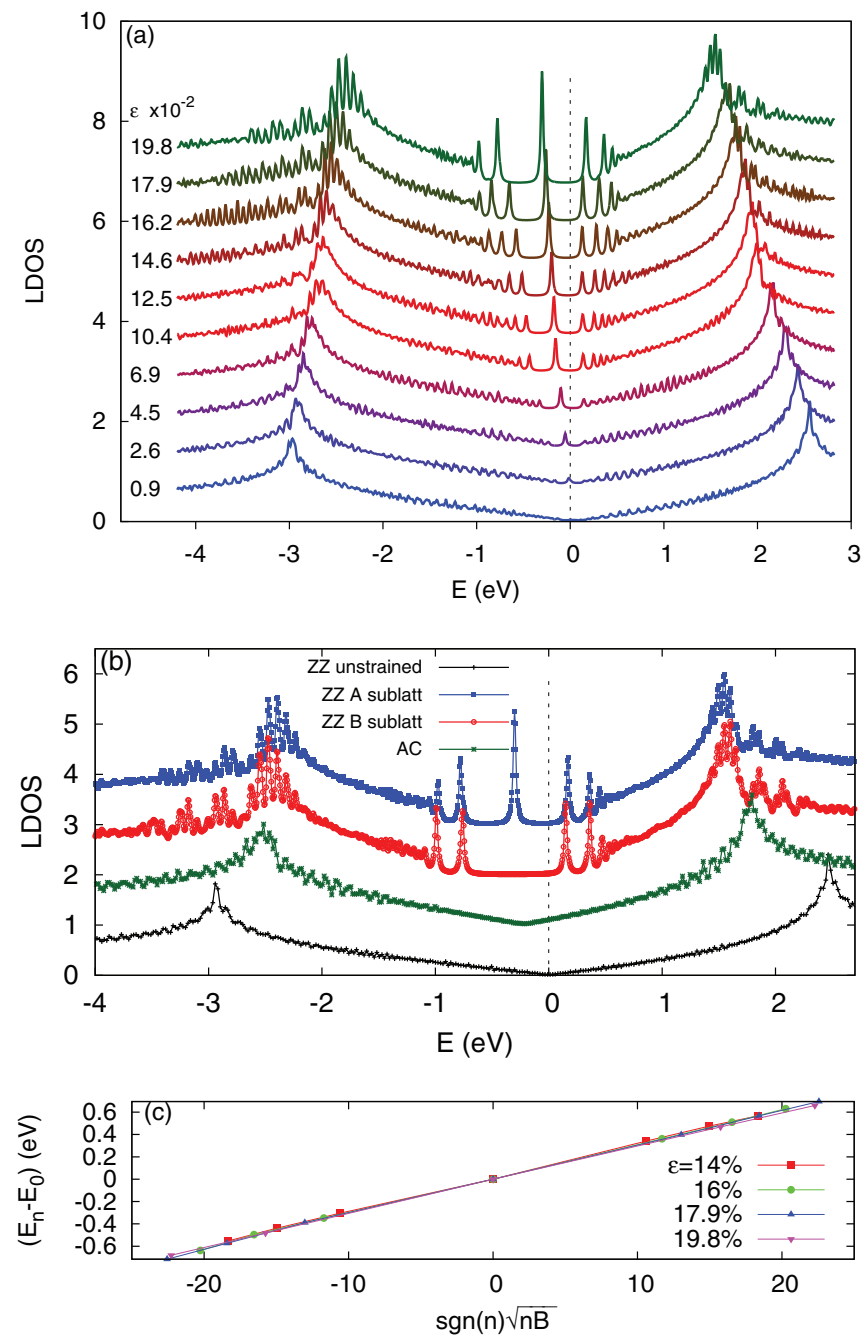

FIG. 6. (Color online) (a) Local density of states in the center of the hexagon for the A sublattice and various strains. (b) Comparison between the central LDOS of the unstrained zigzag flake, strained zigzag flake for both A and B sublattices, and strained armchair flake with strain of $13 \%$. (c) Plot of the positions of the pseudo-Landau levels vs $\operatorname{sgn}(n) \sqrt{n B}$, where $B$ is extracted from the difference between the position of the zeroth and first Landau levels. Note that the energy is shifted such that the Dirac point of the unstrained configuration sits at the Fermi level.

shifts the Dirac point downwards in energy. ${ }^{3}$ In addition, the reduction in the hopping amplitudes shift the van Hove peaks, signaling also a change in the Fermi velocity. Another important effect, noted already in Ref. 6, is the appearance of peaks in the LDOS for the strained zigzag hexagon. These correspond to pseudo-Landau levels generated by the strong pseudomagnetic field observed in the central region of the zigzag hexagon. When compared to the regular Landau levels generated by real magnetic fields, one important difference can be seen: we find that the zeroth Landau level has a finite contribution to the LDOS only in one sublattice, i.e., the sublattice pertaining to the edge atoms under stress. For the nonzero pseudo-Landau levels, the sublattice symmetry still holds. This can be seen in Fig. 6(b) where we show the LDOS in the center of the strained hexagon (with $\epsilon=13 \%$ ) for the A and B sublattices and compare them with the LDOS 


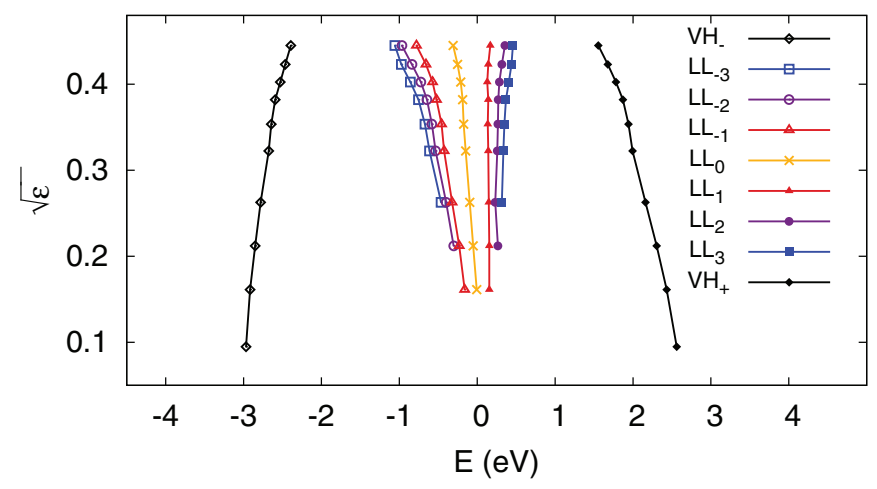

FIG. 7. (Color online) Plot of the energies corresponding to the lower and higher van Hove $(\mathrm{VH})$ singularities and the pseudo-Landau levels (LL), $n= \pm 3, \pm 2, \pm 1,0$, as a function of $\sqrt{\epsilon}$ obtained from the peaks in the LDOS at the center of zigzag strained hexagon.

for the unstrained zigzag case and the LDOS in the center of the strained armchair hexagon. Since the pseudomagnetic field at the center of the armchair hexagon is small, the LDOS does not show pseudo-Landau levels but, instead, exhibits a strain induced shift of both the Dirac points and the van Hove peaks.

The relativistic nature of the pseudo-Landau levels is clearly apparent from Fig. 6(c), where we plot the energy of the pseudo-Landau levels for different strains as a function of $\operatorname{sgn}(n) \sqrt{n B}$. Note that we shift the pseudo-Landau levels such that the zero-Landau level sits at zero energy. Surprisingly, the relationship is linear. Note that the pseudomagnetic field [also shown in Fig. 4(f)] was extracted from the energy gap between the zero and first pseudo-Landau levels by using a constant Fermi velocity and the known relationship: $E_{1}=$

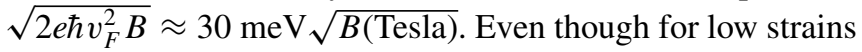
the pseudomagnetic field is proportional to $\epsilon$, the suppression of the Fermi velocity (signaled by the shift of the van Hove peaks) will lead to a nonlinear dependence of the energy of the pseudo-Landau levels on $\sqrt{\epsilon}$. This becomes more drastic at high strains, where the pseudomagnetic field does not follow anymore a linear relationship with respect to $\epsilon$ [see Fig. 4(f)]. This can be seen more clearly in Fig. 7, where the energies of the van Hove peaks and of the pseudo-Landau levels are plotted as a function of $\sqrt{\epsilon}$. Although the spectrum of the unstrained graphene is not particle-hole symmetric, we find that in the zigzag strained graphene samples the higher- $n$ pseudo-Landau levels are symmetric with respect to the zeroth pseudo-Landau level.

Next, we show in Fig. 8 the LDOS maps for various energies for a strained zigzag hexagon with $\epsilon=13 \%$. The contributions to each sublattice are plotted separately for clarity. In panels (a)-(d), we show the LDOS for the strained configuration, while in panels (e)-(h), the LDOS for the unstrained zigzag hexagon is shown for the corresponding energies. The energy of the LDOS map in panel (a) corresponds to the energy of the zeroth Landau level. We observe a large increase in the LDOS in sublattice A (which are under stress at the three edges) in the regions where the pseudomagnetic field is large, together with a suppression of the LDOS in sublattice B. This effect shows the most important difference between pseudomagnetic fields and real magnetic fields. Since a gap appears in the spectrum, and the time-reversal symmetry is not broken, this is a clear

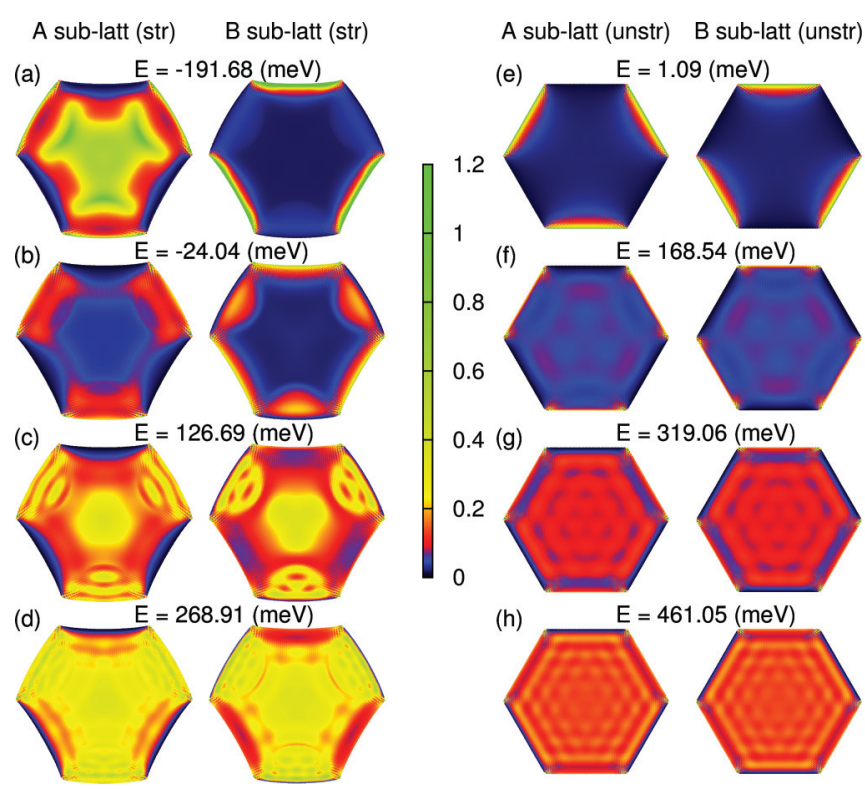

FIG. 8. (Color online) LDOS maps for a strained zigzag hexagon with $\epsilon=13 \%$, (a)-(d), and for an unstrained one, (e)-(h). (a) corresponds to the zero pseudo-Landau level, while (c) and (d) correspond to the first and second pseudo-Landau levels, respectively.

manifestation of a broken sublattice symmetry. If the hexagon would be pulled from the other three sides, the pseudomagnetic field would change sign and the zero pseudo-Landau level will appear in the other sublattice.

The LDOS map shown in Fig. 8(b) corresponds to an energy close to the Dirac point of the unstrained configuration. The only noticeable contributions come from the edgelike states, which are again seen only in sublattice A. The energies of the LDOS maps shown in Figs. 8(c) and 8(d) correspond to the energies of the first and second pseudo-Landau levels, respectively. In this case, we observe that the sublattice symmetry is preserved in the central region of the hexagon. For these energies, the real and pseudo-Landau levels are similar. Interesting features appear also at energies near the van Hove peaks for which the LDOS maps show resonant peaks at the center of the hexagon. At these energies, peaks start developing, while there is no broken sublattice symmetry. It is important to note that the low-energy theory, which relates the strain to a pseudovector potential, is not valid near the van Hove peaks mainly because the dispersion is no longer linear. Movies of the LDOS maps for all energies are presented as Supplemental Material. ${ }^{33}$

Next, we show for comparison in Figs. 8(e)-8(h) the LDOS maps for the unstrained zigzag hexagon. Due to the symmetries of the system, i.e., $\pi / 3$ rotational symmetry, the boundary conditions are such that the wave function is zero in sublattice $\mathrm{A}$ in three nonconsecutive sides and zero in sublattice $\mathrm{B}$ in the other three sides. At the Dirac point, panel (e), edge states with zero energy appear and are located in different sublattices on different sides of the hexagon. For all energies, the LDOS map for the A sublattice is the same as the one for the B sublattice but rotated are $\pi / 3$, as expected due to symmetry arguments.

The LDOS maps for the armchair hexagon are presented in Fig. 9, both for the strained, panels (a)-(d), and the unstrained 


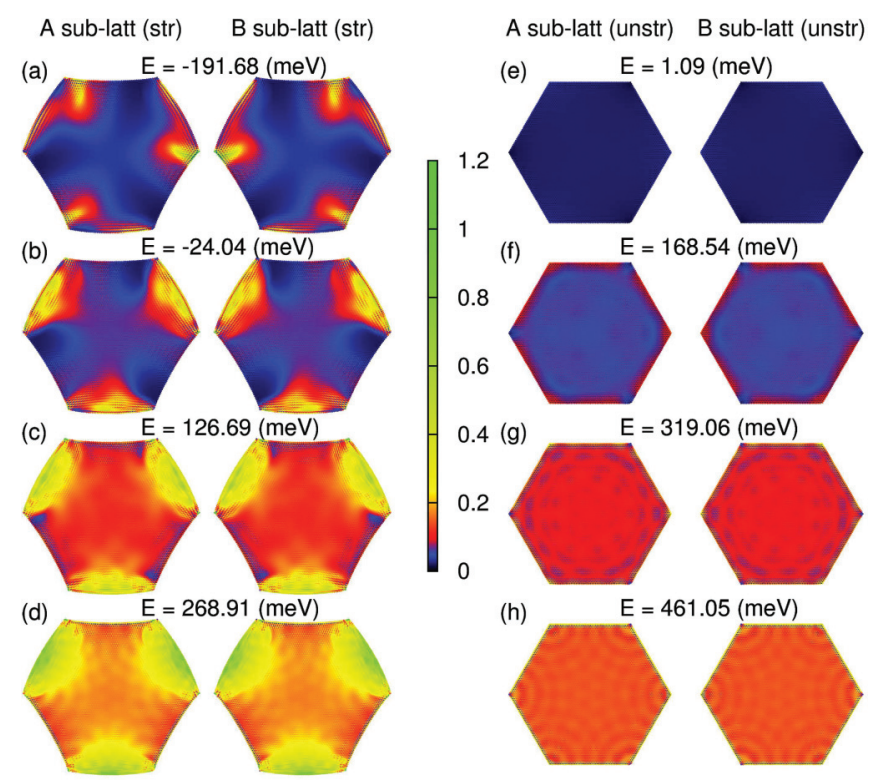

FIG. 9. (Color online) LDOS maps for a strained armchair hexagon with $\epsilon=13 \%$, (a)-(d), and for an unstrained one, (e)-(h).

configurations, panels (e)-(h). The boundary condition now sets the wave function to zero in both sublattices at all hexagon edges. The main symmetry, which is preserved also in the strained configuration, is the mirror symmetry plus a sublattice exchange with respect to $x=0$. The effect of strain on the LDOS maps is not so drastic as the one for the zigzag hexagon. Besides the overall shift in energy due to the appearance of the scalar gauge field, the main modifications can be seen only at the edges of the hexagon where the pseudomagnetic field is large. Since the field changes sign, we see localization of states at the Dirac point in both A and B sublattices.

\section{SUMMARY}

In this paper, we studied the effect of triaxial stress on the electronic and structural properties of hexagonal flakes of graphene with zigzag and armchair edges. We combined molecular dynamics simulations to obtain the relaxed atomic positions and the tight-binding method to describe the electronic properties. We found that lattice deformations under triaxial stress are well described by continuum elasticity theory only for small strains $(\epsilon<15 \%)$ and only in the central part of the sample. The pseudogauge field was found to be neither circular symmetric nor homogeneous in space, i.e., there are modified triangular orbits for zigzag flakes and nonorbital vectors for armchair flakes when the deformed lattice is fully relaxed. The corresponding pseudomagnetic field is nonuniform over the sample and exhibits three fold symmetry. Only for zigzag flakes we find that in the central region, the pseudomagnetic field is large and varies significantly near the edges, while for armchair flakes, the field is very small in the center and oscillating near the edges. The local density of states are completely different at the different sublattices and are mostly affected by the pseudomagnetic field distribution. In the zigzag hexagon, the appearance of pseudo-Landau levels breaks the sublattice symmetry in the zeroth pseudo-Landau level and all relaxed samples show a shift in the energy levels as compared to the undeformed case due to the appearance of a strain induced scalar potential resulting from the expansion of the graphene flake. We find that molecular dynamics relaxation changes strongly the pseudomagnetic field and the local density of states as compared to the deformed nonrelaxed samples from elasticity theory. The latter shows that relaxation of the atomistic structure of the deformed graphene under constraints plays an important role in the electronic properties and that predictions of elasticity theory applicable for continuum sheets should be modified, especially for large strains. Moreover, we found that the field profile cannot be extrapolated from small size to large sizes and there is no specific relation between pseudomagnetic field and the size of systems.

\section{ACKNOWLEDGMENTS}

This work was supported by the EU-Marie Curie IIF postdoctoral Fellowship/299855 (for M.N.-A.), the ESF EuroGRAPHENE project CONGRAN, the Flemish Science Foundation (FWO-Vl) and the Methusalem Funding of the Flemish government.
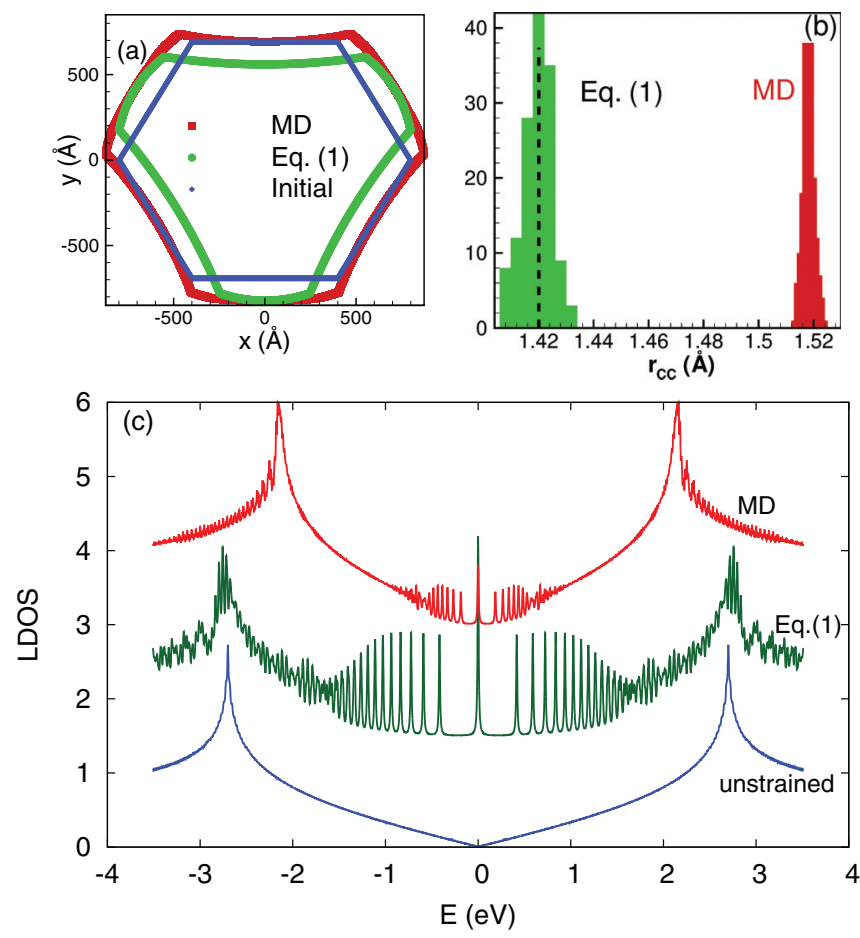

FIG. 10. (Color online) (a) The boundary of the deformed lattice using Eq. (1) (green circles), MD relaxed flake (red squares) and undeformed lattice (blue diamonds) for a hexagonal flake with size $d_{0}=80 \mathrm{~nm}$ subjected to a strain of $17.9 \%$. (b) The C-C bond length histogram in the case of MD relaxation and using Eq. (1) for bonds in a circle with radius $<7 \AA$. (c) Comparison between the central LDOS of the unstrained zigzag flake (blue curve), strained zigzag flake for strain $17.9 \%$ (red line), and that obtained by using deformation based on Eq. (1) (green curve). 


\section{APPENDIX A: EFFECT OF LATTICE RELAXATION}

In this Appendix, we discuss the important effect of the atomistic relaxation of nanoscale samples using bond order force field on the electronic properties of hexagonal graphene flakes. One of the simplest way as to deform the perfect graphene lattice nonuniformly (green diamond in Fig. 10) is by using directly Eq. (1) (here the case of triaxial strain). The boundary of the resulting deformation for hexagonal graphene zigzag flake of size $d_{0}=80 \mathrm{~nm}$ subjected to $17.9 \%$ strain is shown in Fig. 10(a). The corresponding result obtained when using the MD relaxation is shown as red squares in Fig. 10(a). As is obvious from the figure, the MD relaxation expands the area of the flake, thus one expects longer $\mathrm{C}-\mathrm{C}$ bond lengths. In order to show the latter effect, we plotted in Fig. 10(b) the histogram of C-C bond lengths in the central part $(|\mathbf{r}|<7 \AA)$ of the hexagonal flakes for the two mentioned methods. The shorter the bond lengths, the more compact the lattice and the larger the local stress in the central part will be, which results in a larger pseudomagnetic field, see blue triangles and red squares in Fig. 4(f) (see the fields for triaxially stressed unrelaxed hexagonal flake in Ref. 14, which are more than ten times larger than those we report here). In order to reveal the large difference between the pseudomagnetic fields and the consequent effects on the electronic spectrum, we depicted in Fig. 10(c) the LDOS for a system relaxed by MD method for $\epsilon=17.9 \%$, a deformed system with $\epsilon=17.9 \%$ (green curve) by using Eq. (1) and an undeformed hexagonal flake.

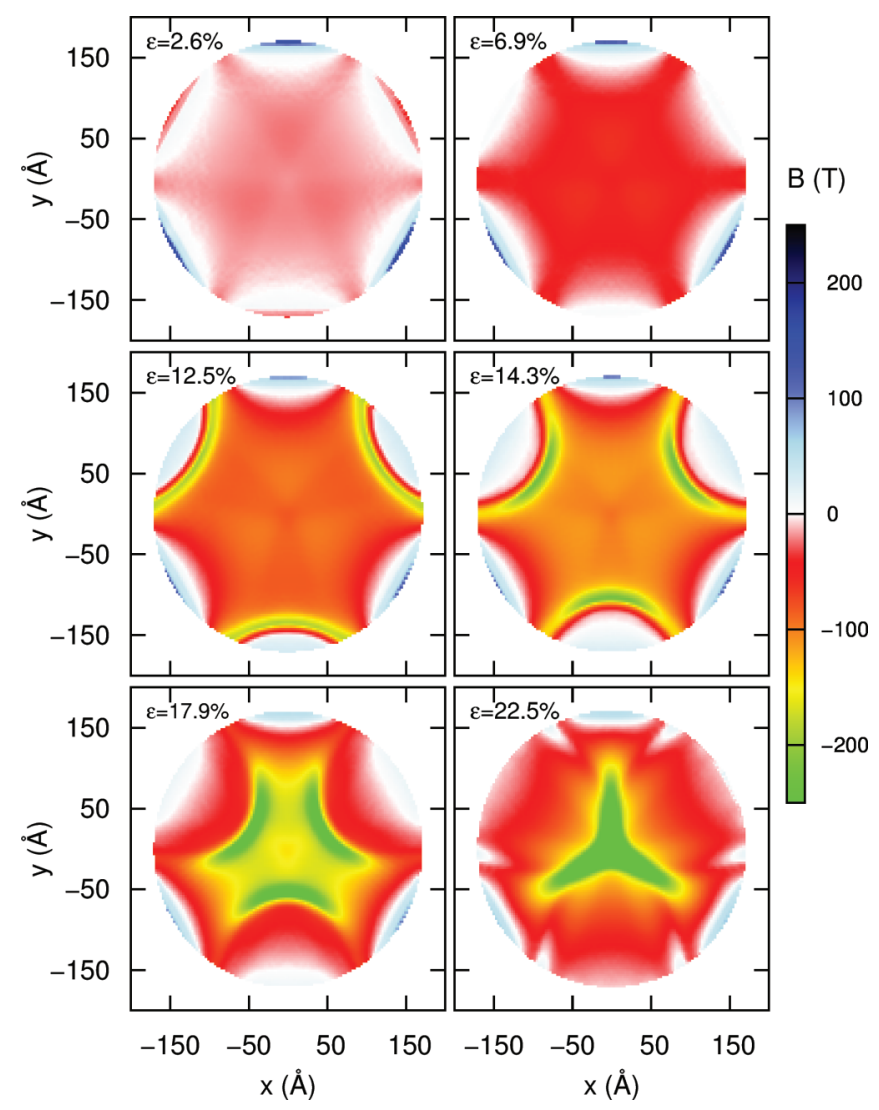

FIG. 11. (Color online) The effect of strain on the pseudomagnetic field in the central part of the zigzag hexagonal graphene flake, $|\mathbf{r}|<17 \mathrm{~nm}$.
Here, we use only the nearest-neighbor hopping amplitudes since the only effect of the next-nearest neighbor term is to shift the Dirac point. The position of the pseudo-Landau levels and the shift of the van Hove peaks are not affected by the change in the next-nearest neighbor hopping. In the case of an undeformed sample [ordinary hexagonal flake shown by blue diamond in Fig. 10(a)], we see that there is no zero energy state at the Dirac point. By deforming the lattice following Eq. (1) with $\epsilon=17.9 \%$ [hexagonal flake shown by green circles in Fig. 10(a)], pseudo-Landau levels with energy separation proportional to $\sqrt{n}$ appear for both electrons and holes. The MD relaxation changes significantly the LDOS profiles because of the mentioned lattice expansion. First, we observe that for the same deformation, $\epsilon=17.9 \%$, the gap between the zero and first pseudo-Landau levels is much smaller, consistent with a much lower pseudomagnetic field. We also find that fewer pseudo-Landau levels can be distinguished, even at these high strains. This could be a consequence of the fact that at high strains the pseudomagnetic field for the MD relaxed system is not constant, and therefore the high- $n$ pseudo-Landau levels are smeared out.

\section{APPENDIX B: EFFECT OF STRAIN ON THE PSEUDOMAGNETIC FIELD PROFILE}

Here, we show the effect of strain on the pseudomagnetic field for different strains in zigzag hexagonal graphene flakes. The larger the strain the larger the pseudomagnetic field. In Fig. 11, we show the pseudomagnetic field for strains in the range of $[2.6 \%-22.5 \%]$ in the central region, i.e., $|\mathbf{r}|<17 \mathrm{~nm}$. It is seen that in the central portion of the zigzag hexagonal graphene flakes, the pseudomagnetic field is not constant, which is in contrast to the continuum elasticity theory prediction and the result obtained from Eq. (1), $B=\frac{16 C \hbar}{a_{0} e}$.

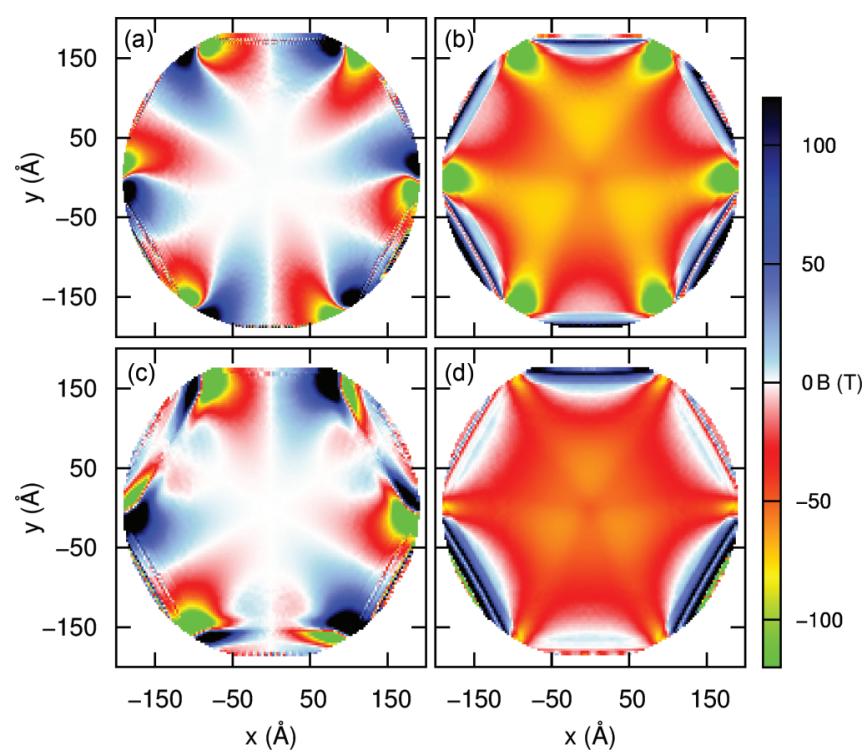

FIG. 12. (Color online) Pseudomagnetic field in the central part of the armchair (a) and (c) and zigzag (b) and (d) hexagonal graphene flake, $|\mathbf{r}|<17 \mathrm{~nm}$ where $\epsilon=6.9 \%$. The fields are obtained either by moving the edges, (a) and (b), or by applying a constant force on the edges, (c) and (d). 


\section{APPENDIX C: ALTERNATIVE METHODS FOR APPLYING STRESS ON THE EDGES}

In addition to the method for applying the stress presented here, one can fix the boundary atoms and shift them gradually in the direction perpendicular to the edges. Since the boundary atoms are not allowed to be relaxed during the simulation, the interatomic distances at the edge remain constant giving high stress at the corners, which consequently affects the stress distribution through the system. The latter effect modifies both the pseudomagnetic field and the LDOS. We found that in case of such a fixed boundary condition the pseudomagnetic field is larger than the one found from the constant applied force method, as shown in Figs. 12(a)-12(d). Moreover, the pseudomagnetic is also more inhomogeneous. The recent experimental realization of triaxial strain in molecular graphene ${ }^{1}$ confirms that the method used in the present work is realistic. The constant motion of the edges was recently used by Qi et al. in order to study resonant tunneling in hexagonal graphene ${ }^{24}$ quantum dots. Their results should be modified in order to have the relevant order of magnitude for the pseudomagnetic field and consequently the correct position of the Landau levels.
${ }^{1}$ K. K. Gomes, W. Mar, W. Ko, F. Guinea, and H. C. Manoharan, Nature (London) 483, 306 (2012).

${ }^{2}$ V. M. Pereira, A. H. Castro Neto, and N. M. R. Peres, Phys. Rev. B 80, 045401 (2009).

${ }^{3}$ A. H. Castro Neto, F. Guinea, N. M. R. Peres, K. S. Novoselov, and A. K. Geim, Rev. Mod. Phys. 81, 109 (2009).

${ }^{4}$ F. Guinea, A. K. Geim, M. I. Katsnelson, and K. S. Novoselov, Phys. Rev. B 81, 035408 (2010).

${ }^{5}$ M. M. Fogler, F. Guinea, and M. I. Katsnelson, Phys. Rev. Lett. 101, 226804 (2008).

${ }^{6}$ F. Guinea, M. I. Katsnelson, and A. K. Geim, Nat. Phys. 6, 30 (2009).

${ }^{7}$ N. Levy, S. A. Burke, K. L. Meaker, M. Panlasigui, A. Zettl, F. Guinea, A. H. C. Neto, and M. F. Crommie, Science 329, 544 (2010).

${ }^{8}$ T. Low and F. Guinea, Nano Lett. 10, 3551 (2010).

${ }^{9}$ M. Vozmediano, M. Katsnelson, and F. Guinea, Phys. Rep. 496, 109 (2010).

${ }^{10}$ M. Yamamoto, O. Pierre-Louis, J. Huang, M. S. Fuhrer, T. L. Einstein, and W. G. Cullen, Phys. Rev. X 2, 041018 (2012).

${ }^{11}$ N. N. Klimov, S. Jung, S. Zhu, T. Li, C. A. Wright, S. D. Solares, D. B. Newell, N. B. Zhitenev, and J. A. Stroscio, Science 336, 1557 (2012).

${ }^{12}$ M. O. Goerbig, J. N. Fuchs, G. Montambaux, and F. Piechon, Phys. Rev. B 78, 045415 (2008).

${ }^{13}$ G. Montambaux, F. Piechon, J. N. Fuchs, and M. O. Goerbig, Phys. Rev. B 80, 153412 (2009).

${ }^{14}$ M. Ramezani Masir, D. Moldovan, and F. M. Peeters, Solid State Commun. (2013), doi: 10.1016/j.ssc.2013.04.001; D. Moldovan, M. Ramezani Masir, and F. M. Peeters, Phys. Rev. B 88, 035446 (2013).

${ }^{15}$ A. Concha and Z. Tešanović, Phys. Rev. B 82, 033413 (2010); A. Raoux, M. Polini, R. Asgari, A. R. Hamilton, R. Fazio, and A. H. MacDonald, ibid. 81, 073407 (2010); F. M. D. Pellegrino, G. G. N. Angilella, and R. Pucci, ibid. 84, 195404 (2011).
${ }^{16}$ M. Neek-Amal and F. M. Peeters, Phys. Rev. B 85, 195445 (2012); 85, 195446 (2012).

${ }^{17}$ A. L. Kitt, V. M. Pereira, A. K. Swan, and B. B. Goldberg, Phys. Rev. B 85, 115432 (2012); 87, 159909(E) (2013).

${ }^{18}$ F. de Juan, M. Sturla, and M. A. H. Vozmediano, Phys. Rev. Lett. 108, 227205 (2012).

${ }^{19}$ F. de Juan, J. L. Manes, and M. A. H Vozmediano, Phys. Rev. B 87, 165131 (2013).

${ }^{20}$ T. L. Linnik, J. Phys.: Condens. Matter 24, 205302 (2012).

${ }^{21}$ M. Neek-Amal, J. Beheshtian, A. Sadeghi, K. H. Michel, and F. M. Peeters, J. Phys. Chem. C 117, 13261 (2013).

${ }^{22}$ H. P. Heiskanen, M. Manninen, and J. Akola, New J. Phys. 10, 103015 (2008); Z. Z. Zhang, K. Chang, and F. M. Peeters, Phys. Rev. B 77, 235411 (2008).

${ }^{23}$ M. Zarenia, A. Chaves, G. A. Farias, and F. M. Peeters, Phys. Rev. B 84, 245403 (2011).

${ }^{24}$ Z. Qi, D. Bahamon, V. Pereira, H. Park, D. Campbell, and A. Castro Neto, Nano Lett. 13, 2692 (2013).

${ }^{25}$ S. J. Stuart, A. B. Tutein, and J. A. Harrison, J. Chem. Phys. 112, 6472 (2000).

${ }^{26}$ D. M. Heyes, Phys. Rev. B 49, 755 (1994).

${ }^{27}$ J. C. Meyer, A. K. Geim, M. I. Katsnelson, K. S. Novoselov, T. J. Booth, and S. Roth, Nature (London) 446, 60 (2007).

${ }^{28}$ L. Covaci and F. M. Peeters, Phys. Rev. B 84, 241401 (2011).

${ }^{29}$ L. Covaci, F. M. Peeters, and M. Berciu, Phys. Rev. Lett. 105, 167006 (2010).

${ }^{30}$ W. A. Muñoz, L. Covaci, and F. M. Peeters, Phys. Rev. B 86, 184505 (2012); 87, 134509 (2013).

${ }^{31}$ M. Neek-Amal, L. Covaci, and F. M. Peeters, Phys. Rev. B 86, 041405 (2012)

${ }^{32}$ J. V. Sloan, Alejandro A. Pacheco Sanjuan, Z. Wang, C. Horvath, and S. Barraza-Lopez, Phys. Rev. B 87, 155436 (2013).

${ }^{33}$ See Supplemental Material at http://link.aps.org/supplemental/ 10.1103/PhysRevB.88.115428 for movies of LDOS maps shown for different energies and different strains. 